\title{
DynaLoc: Real-Time Camera Relocalization from a Single RGB Image in Dynamic Scenes based on an Adaptive Regression Forest
}

\author{
Nam-Duong Duong ${ }^{1}$, Amine Kacete $^{1}$, Catherine Soladie ${ }^{2}$, Pierre-Yves Richard ${ }^{2}$ and Jérôme Royan ${ }^{1}$ \\ ${ }^{1}$ IRT b-com, 1219 Avenue des Champs Blancs, 35510 Cesson-Sévigné, France \\ ${ }^{2}$ IETR/CentraleSupelec, Avenue de la Boulaie, 35576 Cesson-Sévigné, France \\ \{nam-duong.duong, amine.kacete, jerome.royan\}@b-com.com, \{catherine.soladie,pierre-yves.richard $\} @$ centralesupelec.fr
}

Keywords: Camera Relocalization, Adaptive Regression Forest, Hybrid Approach, Single RGB Image

Abstract: Camera relocalization is an important component in localization systems such as augmented reality or robotics when camera tracking loss occurs. It uses models built from known information of a scene. However, these models cannot perform in a dynamic environment which contains moving objects. In this paper, we propose an adaptive regression forest and apply it to our DynaLoc, a real-time camera relocalization approach from a single RGB image in dynamic environments. Our adaptive regression forest is able to fine tune and update continuously itself from evolving data in real-time. This is performed by updating a relevant subset of leaves, which gives uncertain predictions. The results of camera relocalization in dynamic scenes report that our method is able to address a large number of moving objects or a whole scene to gradually change in order to obtain high accuracy avoiding accumulation of error. Moreover, our method achieves results as accurate as the best state-of-the-art methods on static scenes dataset.

\section{Introduction}

Camera pose estimation is a key feature of Augmented Reality (AR), robot navigation and autonomous vehicles (self-driving), which requires an accurate tracking of camera viewpoint in a 3D space. The main solution of camera pose estimation for commercial systems is known as Simultaneously Localization And Mapping (SLAM) (Davison et al., 2007; Klein and Murray, 2007; Mur-Artal et al., 2015; Engel et al., 2014; Tateno et al., 2017). SLAM processes an ordered sequence of images. In the case of fast camera motion or sudden change of viewpoint such as with a hand-held camera, tracking failure interrupts camera pose estimation. When this happens, camera relocalization is needed to retrieve camera pose after tracking lost, rather than restarting the localization again from scratch. However, the existing camera relocalization methods in SLAM need to store a large set of key-points or key-frames. Consequently, memory usage as well as processing time increase with respect to the size of models.

Recently, machine learning approaches for camera relocalization have appeared to tackle these constraints. These methods (Kendall et al., 2015; Kendall and Cipolla, 2016; Walch et al., 2017; Clark et al., 2017) can estimate camera pose in real-time from each image. However, limitations of these methods lie in their moderate accuracy and the lack of confidence score for each pose estimation. To improve the accuracy as well as address uncertainty prediction in deep learning approaches, (Shotton et al., 2013; Guzman-Rivera et al., 2014; Valentin et al., 2015; Brachmann et al., 2016; Duong et al., 2018) presented hybrid methods using random forest and geometric algorithms for camera relocalization with higher accuracy. Such methods are robust to illumination changes and partial occlusion. Yet, these machine learning based methods and hybrid methods still fail to challenge dynamic scenes with moving objects.

Moving objects are often encountered in many augmented reality scenarios such as maintenance, assembly and quality control task for Manufacturing and Construction application where: devices are equipped only with a single RGB camera, scenes are changing gradually over time, tasks requires workbench scale 3D registration. Dynamic data is not a challenge dedicated to camera relocalization. But it also appears in many other applications using machine learning. Indeed, for machine learning in general, a model is learned from a set of training data. If data is changing and the learned model is not able to update itself, this model is no longer accurate. Thus, these methods require an adaptive learning 
model which has the capacity to update rapidly to the changes of data in order to maintain the accuracy of the learned model.

In this paper, we focus on handling challenges of dynamic data for generic machine learning as well as for camera relocalization. Our contributions are divided into two parts as follows.

Our first contribution is to propose an online updating regression forest process. We call it Adaptive Regression Forest - ARF. The main idea is that the ARF updates instantly a subset of leaves which gives uncertain predictions. It is performed by two main originalities based on detection and update of uncertain leaves.

The first originality of our ARF is to detect the leaves giving uncertain predictions. There are two criteria for a leaf to become a passive leaf: having a high variance of predictive model; giving repeatedly a result rather different from the other leaves.

The second originality of our ARF is to update in real time passive leaves of the regression forest model. This is performed by re-modeling their predictive model from new computed labels. These labels are computed based on results given by the other leaves (actives leaves). Note that the update is only performed on passive leaves and not on the whole regression forest model. This process leads to select relevant data to update efficiently at runtime the model.

The second contribution is to present our DynaLoc. It is a real time camera relocalization in dynamic scenes based on the generic ARF. For this application, the ARF predicts 3D points in the world coordinates system which correspond to $2 \mathrm{D}$ points in the image. The originality is to keep the structure of the forest (trees and nodes) by using invariant SURF feature at split nodes as proposed in (Duong et al., 2018).

A last contribution concerns the creation of a public database to evaluate camera relocalization methods. It is the first public database including dynamic scenes, which is one of the most important challenge for (re)localization methods.

In the following sections, we first present the related work in section 2. Our adaptive regression forest is described in section 3. Section 4 presents our DynaLoc that is a real-time camera relocalization in dynamic scenes based on the adaptive regression forest. Section 5 shows and discusses our results on rigid scenes dataset and on our dynamic scenes dataset. Finally, section 6 provides some conclusions and perspectives.

\section{Related Work}

In this section, we first present camera localization (SLAM) methods in dynamic environment. Then, we introduce state-of-the-art camera relocalization methods and their limit regarding dynamic scenes.

\subsection{SLAM in dynamic environment}

Dynamic environment with moving objects is still known as a difficult challenge in camera localization community. The most typical methods to address this challenge are RANSAC algorithms. RANSAC considers data on moving objects as outliers in order to eliminate them in camera pose estimation process. However, if a large number of objects move, the number of outliers will be superior to the number of inliers. In this case, RANSAC algorithm processes incorrectly. In recent years, several SLAM methods focus on this specific issue of dynamic scenes.

Most works perform detection and segmentation of moving objects in each frame. This is to avoid their influence on the camera pose estimation. (Wangsiripitak and Murray, 2009; Riazuelo et al., 2017; Bescos et al., 2018) detect and track the known dynamic objects or movable objects e.g. people, vehicles. (Sun et al., 2017; Alcantarilla et al., 2012) detect moving objects by using optical flow between consecutive frames. Nevertheless, these methods try to remove moving objects to compute camera pose. Hence, they cannot process in scenes where the whole scene changes gradually.

To overcome this, (Newcombe et al., 2015) proposed a real-time dense dynamic scene reconstruction and tracking based on depth images by using a single rigid volumetric TSDF (Truncated Signed Distance Function). This method requires a RGB-D camera to robustly detect moving objects based on ICP (Iterative Closest Point) algorithm, which is not our application case. (Tan et al., 2013) proposes another SLAM approach that can detect any changes by projecting the map feature into the current frame. In real-time, it can update the $3 \mathrm{D}$ points cloud and keyframes stored in a memory to add new elements or remove those which do not exist anymore. This allows to address the challenge of whole scene changing gradually. Yet, it has the limitations of geometric approaches, that we detail below.

\subsection{Camera relocalization methods}

The camera relocalization methods can be classified into three different groups, namely: geometric 
approaches; deep learning approaches; hybrid approaches.

Geometric approaches are methods that use geometric point correspondences in order to calculate camera pose. These methods first extract a set of sparse features (Sattler et al., 2011; Baatz et al., 2012; Sattler et al., 2017) or dense features (Newcombe et al., 2011; Engel et al., 2014; Glocker et al., 2015) from each frame. Then, these features are matched with keypoints of a scene model. Eventually, camera pose is determined by minimizing feature re-projection error. These methods require a scene model that includes a set of keypoints attached to feature vectors and 3D positions in the world coordinates. This model is constructed from a set of images of the scene by performing Structure from Motion (SfM) (Sattler et al., 2011; Baatz et al., 2012; Sattler et al., 2017) or encoding a set of keyframes (Mur-Artal et al., 2015; Glocker et al., 2015). Most of these geometric methods attempt to reduce the computational complexity of feature matching. (Sattler et al., 2011; Sattler et al., 2017) use a visual vocabulary for efficient 2D-to-3D matching. Even so, since the matching time depends on the size of the model, this reduces considerably the scalability.

Deep learning approaches in camera relocalization are usually considered as a supervised regression problem. A regression model is learned from the labeled data (images with their 6-DoF camera poses) of the known scenes. (Kendall et al., 2015) was the first to propose the use of deep learning as an end-toend camera pose estimation approach. (Kendall and Cipolla, 2016) generates a probabilistic pose estimation by using dropout after every convolutional layer. (Walch et al., 2017) is an improvement of PoseNet's architecture with spatial Long Short Term Memory (LSTM) added after CNN layers, that aims at reducing the dimension of the feature vector, thus providing important improvements in localization performance. (Kendall and Cipolla, 2017) solves an ambiguity of scale factor between location error and orientation error in the loss function of (Kendall et al., 2015) by a novel loss function based on re-projection error. (Clark et al., 2017) exploits the temporal information by using a LSTM to predict camera pose based on multiple consecutive frames. However, all these methods exhibit low relocalization accuracy so far.

Hybrid approaches estimate camera pose by fusing both machine learning approaches and geometric approaches. Machine learning part is applied to learn and predict $3 \mathrm{D}$ position of each pixel in world coordinates instead of 6-DoF camera pose as presented in deep learning approaches above. Then, geometric part infers camera pose from these correspondences.
The first hybrid methods are based on the regression forest to fast define 2D-3D point (Brachmann et al., 2016) or 3D-3D point (Shotton et al., 2013; Guzman-Rivera et al., 2014; Valentin et al., 2015; Meng et al., 2017) correspondences. (Brachmann et al., 2017; Brachmann and Rother, 2018), instead, uses a deep learning architecture to predict scene coordinates. (Duong et al., 2018) proposes a sparse feature regression forest learning with a novel split function aiming at accelerating computational time and keeping high accuracy. (Cavallari et al., 2017) proposes an on-the-fly training camera relocalization from RGB-D images which deals with how to accelerate learning a new scene. It adapts a pre-trained forest to a new scene by retaining the internal nodes. Yet, it needs to update all the leaves with the ground truth of this new scene.

However, all camera relocalization methods mentioned above are not suitable for dynamic scenes with moving objects. Indeed, geometrical model or learned model are built from rigid scenes. Therefore, when some objects move, these models are no longer accurate to infer camera pose. To overcome this challenge, we expand the real-time accurate hybrid method based on the regression forest (Duong et al., 2018) to our ARF method. The ARF model is able to update only a subset of uncertain leaves to quickly modify without any interruption the trained model according to changes of the scene such as moving objects.

\section{Adaptive Regression Forest}

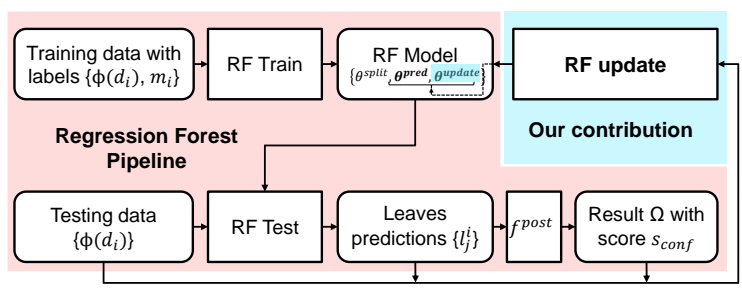

Figure 1: Adaptive regression forest pipeline. The common regression forest method (red components) is extended with an online update step (blue component). It refines predictive model in real-time from new computed labels.

In this section, we first introduce the common regression forest pipeline which our method is based on. Then, we explain the limitations of this pipeline and how we overcome them by using our ARF method. Finally, we detail our ARF methodology including: adaptive leaf; passive leaves detection and passive leaves update. 


\subsection{Regression Forest pipeline}

Fig. 1 presents the common regression forest pipeline (components in red) including two phases: training and testing. A regression forest is a set of $N$ decision trees $\mathcal{F}=\left\{\mathcal{T}_{j}\right\}$. Each tree $\mathcal{T}_{j}=\left\{\theta^{\text {split }}, \theta^{\text {pred }}\right\}$ consists of split functions $\theta^{\text {split }}$ at internal nodes and predictive models $\theta^{\text {pred }}$ at leaf nodes. These parameters are learned from a set of labeled data $\left\{\phi\left(d_{i}\right), m_{i}\right\}$, where $\phi\left(d_{i}\right)$ is the feature vector extracted from data $d_{i}$ with the label $m_{i}$. The split function $\theta^{\text {split }}$ is a weak learner. It is used to split a subset of data into left child node and right child node. The weak learner is trained to maximize an objective function aiming to reduce variance. The training terminates when the tree reaches a maximum depth or when a node has few data. The predictive model at each leaf node is represented by a distribution $\theta^{\text {pred }}=\mathcal{N}\left(m, \bar{m}, \Sigma_{m}\right)$. It is computed from a set of labels $m$ of data reaching this leaf. $\bar{m}$ and $\Sigma_{m}$ are respectively the mean and the covariance of the Gaussian distribution.

For testing phase, each testing data that is represented by a set of features $\left\{\phi\left(d_{i}\right)\right\}$ passes through the regression forest model to obtain multiple leaves predictions $\left\{l_{j}^{i}\right\}$, in which $l_{j}^{i}$ is a prediction of the decision tree $j$ for the feature $\phi\left(d_{i}\right)$. All predictions are combined to compute the final output result $\Omega$ with a confidence score $s_{\text {conf }}$ by using a post-processing function $f^{\text {post }}$.

\subsection{Limitations of Regression Forest}

In the regression forest, leaves with a high variance are not informative. They make noisy predictions. Thus, all leaves whose variance is greater than a threshold $T_{v a r}$ are discarded to eliminate noise and improve accuracy. This leads to the fact that a subset of leaves are stored in the regression forest, but they are never used.

Another challenge of regression forest and of machine learning methods in general is facing dynamic data. Because a regression model is learned from static data, the pretrained regression model will be no longer accurate if some data change. In this case, it requires re-training from scratch a whole model with a redefinition of data labels.

\subsection{Methodology}

To overcome the limitations of the regression forest, we propose an adaptive regression forest. It is an extension of the common regression forest pipeline by adding an update step, as shown in Fig. 1 (in blue). At the beginning, we assume that a regression forest is learned from initial training data. Then, in runtime, their labels change. The update step of our ARF improves consecutively the accuracy of the regression forest model. It adapts robustly to dynamic data as well as refines unused leaves (see 3.2). This is performed by updating leaf nodes based on input data $\left\{d_{i}\right\}$, predictions $\left\{l_{j}^{i}\right\}$ and final output result $\Omega$.

In this section, we first give the definitions of our ARF parameters. Then, we describe the two main steps of our update process: passive leaves detection and passive leaves update. This ARF update is detailed in Fig. 2.

\subsubsection{Definitions of ARF parameters}

Several concepts are used in our ARF: active/passive leaf, good/bad prediction, validation function, confidence score. We present them in this subsection.

The parameters of each tree of a regression forest is extended to the ARF model $\left\{\theta^{\text {split }}, \theta^{\text {pred }}, \theta^{\text {update }}\right\}$, where $\theta^{\text {update }}=\left\{s, n_{\text {false }}, S_{\text {data }}\right\}$ are the parameters used in the update process.

$s$ denotes the status of a leaf: $s \in\{0$ : passive, 1 : active $\}$. A passive leaf is a leaf whose prediction is not accurate, see 3.3.2 for more details. We call it passive because those leaves are not used during the final process that use the random forest prediction. In contrary, predictions which come from active leaves are used to estimate final result. Therefore, the postprocessing function in our ARF is defined as follows:

$$
\Omega=f^{\text {post }}\left(\left\{d_{i}, l_{j}^{i} \mid s_{j}^{i}=1\right\}\right)
$$

Where $s_{j}^{i}$ is the status of the leaf of the tree $j$ corresponding to the feature vector $\phi\left(d_{i}\right)$.

$n_{\text {false }}$ denotes the number of consecutive times an active leaf gives a bad prediction (see 3.3.2). $S_{\text {data }}$ is a stack of data that stores the data $d_{i}$ and the final result $\Omega$ at each passive leaf. We need it for passive leaves update (see 3.3.3).

We introduce a validation function $f^{v a l}$ to define good and bad predictions.

$$
f^{\text {val }}\left(f^{\text {err }}\left(\Omega, d_{i}, l_{j}^{i}\right)\right)= \begin{cases}1, & f^{\text {err }}(.)<T_{\text {val }}, \text { good } \\ 0, & \text { otherwise, bad }\end{cases}
$$

Where $f^{e r r}\left(\Omega, d_{i}, l_{j}^{i}\right)$ denotes an error function of each leaf prediction $l_{j}^{i}$ based on the output $\Omega$ and input data $d_{i} . T_{v a l}$ is an error threshold in order to determine good/bad predictions.

After the validation step, we obtain $n_{\text {good }}$ and $n_{\text {bad }}$ predictions. A score $s_{\text {conf }}$ is calculated to evaluate the confidence score of final result:

$$
s_{\text {conf }}=\frac{n_{\text {good }}}{n_{\text {good }}+n_{\text {bad }}}
$$




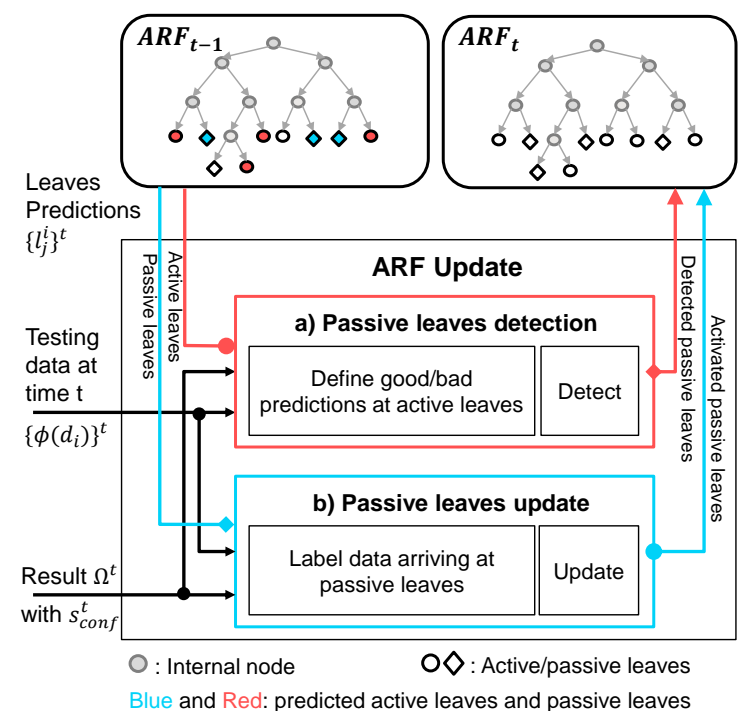

Figure 2: Adaptive regression forest update process. The predictive models at leaf nodes evolve by part over time without training from scratch a whole new model. ARF performs simultaneously two steps: passive leaves detection and passive leaves update.

If this confidence score is greater than $T_{\text {conf }}$, the update of ARF will be proceeded based on the output result $\Omega$. $T_{\text {conf }}$ is a confidence threshold to ensure that the output result is reliable. It aims at limiting accumulation of errors during the update process.

\subsubsection{Passive leaves detection}

Passive leaves detection aims at detecting leaves being no longer relevant and change their status to passive. After the initial training phase, the status of each leaf is defined by:

$$
s= \begin{cases}1, \text { active, } & \operatorname{tr}\left(\Sigma_{m}\right)<T_{\text {var }} \\ 0, \text { passive, } & \text { otherwise }\end{cases}
$$

Where $\operatorname{tr}\left(\Sigma_{m}\right)$ is the trace of the covariance matrix $\Sigma_{m}$, $T_{\text {var }}$ is a threshold to define passive leaves. In the testing phase, an active leaf becomes a passive leaf, when it gives consecutively uncertain results. Fig. 2-a) illustrates the passive leaves detection. We first define good/bad predictions from a set of active leaves predictions based on the validation function (2). We then use $n_{\text {false }}$ to count the number of consecutive times an active leaf is considered as a bad prediction. Finally, if $n_{\text {false }}>T_{\text {false }}$, this active leaf becomes a passive leaf and its status is assigned to 0 (passive). $T_{\text {false }}$ is a detection threshold to ensure that this leaf is really an uncertain leaf. $n_{\text {false }}$ is reassigned to 0 as soon as the active leaf gives a good prediction once. This aims at avoiding mistakes of determining good/bad prediction from the error function (2) due to noisy data.

\subsubsection{Passive leaves update}

The passive leaves update aims at remodeling the predictive models of passive leaves from new estimated labels. These new labels are calculated based on the estimated result $\Omega$ and input data $\left\{d_{i}\right\}$. Fig. 2-b) shows the passive leaves update step. It only processes at predicted passive leaves. When a feature $\phi\left(d_{i}\right)$ passes through our forest and terminates in a passive leaf, we firstly collect the corresponding input data $d_{i}$ and the result $\Omega$ in a stack of data $S_{d a t a}$ associated to this passive leaf. These elements stored in the stack of data allow to compute new labels of data. When the number of data in the stack at a passive leaf is large enough $\left|S_{\text {data }}\right|>T_{\text {data }}$ ( $T_{\text {data }}$ is a threshold to ensure that the number of data is sufficient to learn a new distribution), we calculate labels $m_{i}$ of $d_{i}$ by using a labeling function:

$$
m_{i}=f^{\text {label }}\left(S_{\text {data }}\right)
$$

And then a Gaussian distribution of this leaf is modeled from these labels $\left\{m_{i}\right\}$. Finally, the status of this leaf is defined by the function (4). The stack of data $S_{\text {data }}$ is reset. The passive leaves update and passive leaves detection are performed at the same time to accelerate ARF system.

\section{Camera Relocalization in Dynamic Scenes}

In this section, we introduce our DynaLoc, a realtime camera relocalization method from only RGB images in dynamic scenes. Inspired by (Duong et al., 2018), we propose a hybrid method merging our ARF (described in the section 3) and geometric methods. Our ARF is applied to learn and predict 2D-3D point correspondences. The geometric part uses PnP and RANSAC algorithms in order to compute camera pose from these correspondences. Fig. 3 illustrates our DynaLoc pipeline. Our method is summarized in three principal steps. Firstly, we present how to initially train the ARF from RGB images. Then, camera pose estimation is performed based on the ARF and geometric algorithms. Finally, we detail the online ARF update process for camera relocalization.

\subsection{Initial ARF training}

The ARF is initialized according to the training step detailed in (Duong et al., 2018). An ARF is learned 


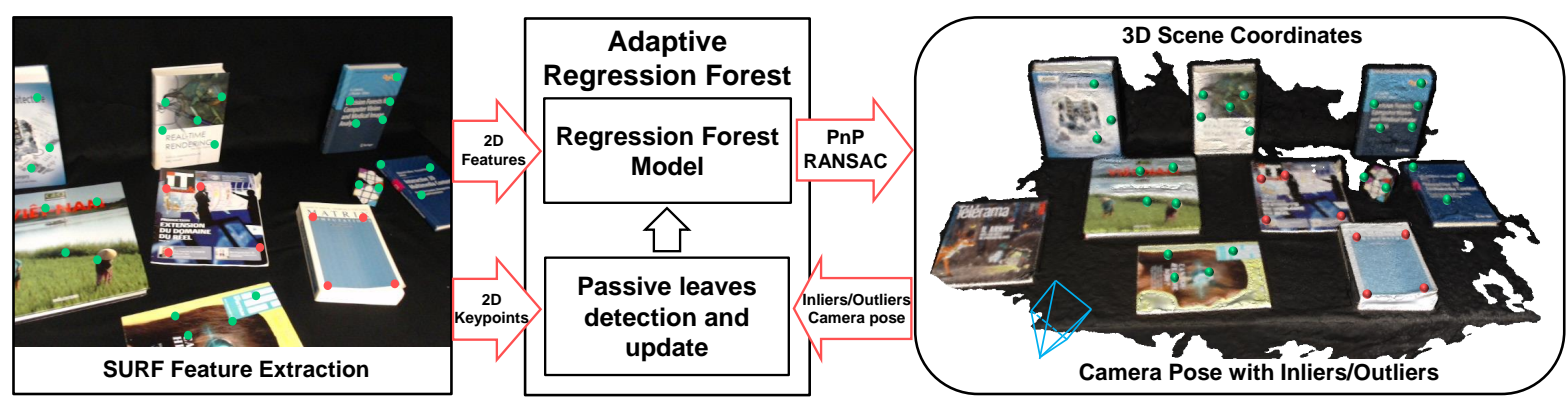

Figure 3: DynaLoc: Real-time camera relocalization in dynamic scenes based on the ARF. We apply the ARF to hybrid camera relocalization: from a set of SURF feature detected on each RGB image, the ARF predicts a set of 3D world coordinates. They correspond to active leaves (green and red points). Then PnP and RANSAC algorithms determine inliers (green points) to estimate camera pose and reject outliers (red points). Finally, if the camera pose is precisely estimated, the ARF is updated by using the estimated camera poses, inliers, outliers and 2D positions of keypoints.

from a set of labeled feature vectors $\left\{\phi\left(d_{i}\right), m_{i}\right\}$ which are extracted from a set of RGB training images. $\phi\left(d_{i}\right)$ is a SURF feature vector extracted around a $2 \mathrm{D}$ keypoint position $d_{i} . m_{i}$ is the 3D point in the world coordinates system corresponding to $d_{i}$. The label $m_{i}$ is defined by running triangulation algorithm (Hartley and Zisserman, 2005) for each pair of matching keypoints $\left(d_{k}, d_{l}\right)$ of two RGB images, whose poses $\left(\Omega_{k}, \Omega_{l}\right)$ are supposed to be known in advance by using a localization system (marker based, 3D model based, SLAM, tracking, etc.):

$$
\left\{\begin{array}{l}
d_{k} \times\left(K \Omega_{k}^{-1} m_{i}\right)=0 \\
d_{l} \times\left(K \Omega_{l}^{-1} m_{i}\right)=0
\end{array}\right.
$$

Where $K$ is the matrix of the camera intrinsic parameters.

Each tree of ARF is initially trained by a random subset of data to determine the split functions. We use whole SURF feature vector as proposed in (Duong et al., 2018) and adaptive leaf nodes $\left\{\theta^{\text {split }}, \theta^{\text {pred }}, \theta^{\text {update }}\right\}$ (see 3.3). Each leaf node stores the $3 \mathrm{D}$ positions corresponding to a set of SURF features reaching it which is represented by a Gaussian distribution $\mathcal{N}\left(m, \bar{m}, \Sigma_{m}\right)$. The status $s$ of each leaf of ARF is defined by the status definition function (4). $n_{\text {false }}$ is assigned to 0 and $S_{\text {data }}$ is initialized by an empty set.

\subsection{Camera pose estimation}

Firstly, a set of SURF keypoints and features $\left\{d_{i}, \phi\left(d_{i}\right)\right\}$ is extracted from each RGB input image. They pass through the ARF $\left\{\mathcal{T}_{j}\right\}$ to achieve a set of predictions $\left\{l_{j}^{i}\right\}$ that contains $3 \mathrm{D}$ world coordinates predictions $\left\{\hat{m}_{i}\right\}$ corresponding to 2D SURF keypoints $\left\{d_{i}\right\}$. All 2D-3D correspondences coming from active leaves are used to estimate camera pose based on the post-processing function (1). In camera relocalization, $f^{\text {post }}$ function of ARF is defined by $\mathrm{PnP}$ and RANSAC functions in order to remove bad predictions (outliers) and keep good predictions (inliers). RANSAC generates a set of hypothetical poses $\left\{\Omega_{i}\right\}$ by performing PnP on random subsets of $2 \mathrm{D}-3 \mathrm{D}$ point correspondences. The best inliers are defined by maximizing the number of inliers corresponding to each hypothesis based on the validation function (2), in which the error function $f^{e r r}($.$) is defined as a$ re-projection error function:

$$
f^{e r r}\left(\Omega, d_{i}, l_{j}^{i}\right)=\left\|d_{i}-K \Omega_{i}^{-1} \hat{m}_{i}\right\|^{2}
$$

The final camera pose $\Omega$ is carried out by running $\mathrm{PnP}$ once on all inliers to minimize the sum of reprojection error.

\subsection{Online ARF update}

The ARF in our DynaLoc keeps the internal nodes and the structures of the trees. It only updates continuously predictive models at uncertain leaf nodes to adapt to the changes in dynamic scenes. The update process is based on two main steps: passive leaves detection and passive leaves update, as described in section 3.3.

In the passive leaves detection (see 3.3.2), the good and bad predictions correspond to inliers and outliers respectively which are defined in section 4.2. In the passive leaves update (see 3.3.3), the stack $S_{\text {data }}$ of each passive leaf collects constantly 2D positions of SURF keypoints and camera poses $\left(d_{i}, \Omega_{i}\right)$. The labeling function (5) is defined by the triangulation algorithm (Hartley and Zisserman, 2005). From a pair of data in the stack $\left(d_{k}, \Omega_{k}\right)$ and $\left(d_{l}, \Omega_{l}\right)$, the label $m_{i}$ is computed based on the triangulation function (6). Thus, a set of $T_{\text {data }}$ data at each passive leaf defines a set $\left\{m_{i}\right\}$ of $\frac{T_{\text {data }} \cdot\left(T_{\text {data }}-1\right)}{2 D}$ points. A new Gaussian distribution $\mathcal{N}\left(m, \bar{m}, \Sigma_{m}\right)$ is modeled based on 


\begin{tabular}{|c|c|c|c|c|c|c|c|c|c|c|c|c|}
\hline \multirow{3}{*}{ Infos } & \multicolumn{3}{|c|}{ DynaScene-01 } & \multicolumn{3}{|c|}{ DynaScene-02 } & \multicolumn{3}{|c|}{ DynaScene-03 } & \multicolumn{3}{|c|}{ DynaScene-04 } \\
\hline & Train & & & Train & & & Train & & & Train & & \\
\hline & Seq-00 & Seq-01 & Seq-02 & Seq-00 & Seq-01 & Seq-02 & Seq-00 & Seq-01 & Seq-02 & Seq-00 & Seq-01 & Seq-02 \\
\hline \#Frames & 669 & 681 & 681 & 831 & 1136 & 1075 & 743 & 842 & 1140 & 958 & 1395 & 2109 \\
\hline Occlusion & No & No & No & No & Yes & No & No & No & No & No & Yes & Yes \\
\hline Illumination change & No & No & No & No & No & No & No & Yes & No & No & Yes & Yes \\
\hline Moving objects & $0 \%$ & $0 \%$ & $30 \%$ & $0 \%$ & $0 \%$ & $60 \%$ & $0 \%$ & $0 \%$ & $100 \%$ & $0 \%$ & $0 \%$ & $100 \%$ \\
\hline
\end{tabular}

Table 1: DynaScenes dataset. A RGB images dataset is used to evaluate camera relocalization methods in dynamic scenes.

3D points. The function (4) validates the status of new leaf model.

A key feature of this update process is that the function (4) can eliminate the new model and reset the data buffer at leaf nodes when the variance is too high (outliers). Thus, changes of the scene such as moving/coming objects or wrong camera pose estimation, generating inconsistent 3D points during triangulation step, will not damage the model. Nevertheless, when a moving object will become stationary again, the triangulation function will estimate correct labels, and the variance will become low enough to reactivate the leaf. Thus, the model is updated to remain accurate when the scene has changed.

\section{Experiments}

In this section, we first present the datasets used in our experiments. Especially, we introduce our dataset including dynamic scenes, we call it DynaScenes dataset. Then, we demonstrate the usefulness of our ARF on both static and dynamic scenes by comparing it with a regression forest (RF) approach. Finally, we compare our DynaLoc with state-of-the-art RGB camera relocalization methods.

\subsection{Datasets}

We evaluate our method on two datasets, both static and dynamic scenes, to compare with state-of-the-art methods. These datasets are indoor scenes. Each one provides intrinsic matrix of camera, thousands of images of different trajectories at $640 \times 480$ resolution and annotations (camera pose for each frame).

The 7-scenes dataset (Shotton et al., 2013) contains seven static scenes in room-scale. Each scene includes some RGB-D image sequences which are captured around a single room and annotated by using Kinect Fusion (Newcombe et al., 2011). The data is challenging with texture-less surface, repeated structure or fast movement of the camera, that provides many blurry images.
The DynaScenes dataset ${ }^{1}$ is a completely new dataset for camera relocalization proposing dynamic environments. To create challenging dynamic scenes with gradually moving objects, we simulates real scenarios as augmented reality based maintenance on a workbench where users teleport sequentially objects from one location to another. We also inject challenges of illumination changes and occlusion into this dataset. This dataset consists of four scenes. Each one contains three absolutely different RGB image sequences of the same scene (one training sequence and two testing sequences). More detail characteristics of this dataset are given in Tab. 1. The ground truth of camera pose is accurately acquired by using a HTC Vive tracker rigidly attached on the camera. The camera can move around within a visible area of two lighthouse base stations. This allows us to continuously track the orientation and trajectory of the tracker. A rigid transformation matrix between tracker pose and camera pose is estimated by using the hand-eye calibration method (Tsai and Lenz, 1988). Camera pose is found based on tracker pose and this transformation matrix. We perform a synchronization to get image frame and tracker pose at the same time.

\subsection{ARF versus RF}

ARF performance on dynamic scenes. Fig. 4 shows the performance of our ARF compared to a regression forest with chosen leaves (RF) on a dynamic sequence which contains objects moving gradually. The results demonstrate that when objects are static, both approaches achieve high accurate localization as shown at beginning of Fig. 4-a,b). As soon as more than $30 \%$ of objects move, the RF approach has large error because the number of inliers reduces rapidly. On the other hand, the accuracy of our DynaLoc is maintained thanks to the ARF update process. Fig. 4d) gives the evolution of the number of active leaves over time. When some objects move, the corresponding active leaves are defined as passive leaves and the percentage of active leaves drops. When these objects return to a static state, this percentage increases

\footnotetext{
${ }^{1}$ https://github.com/duongnamduong/DynaScenes-
} 


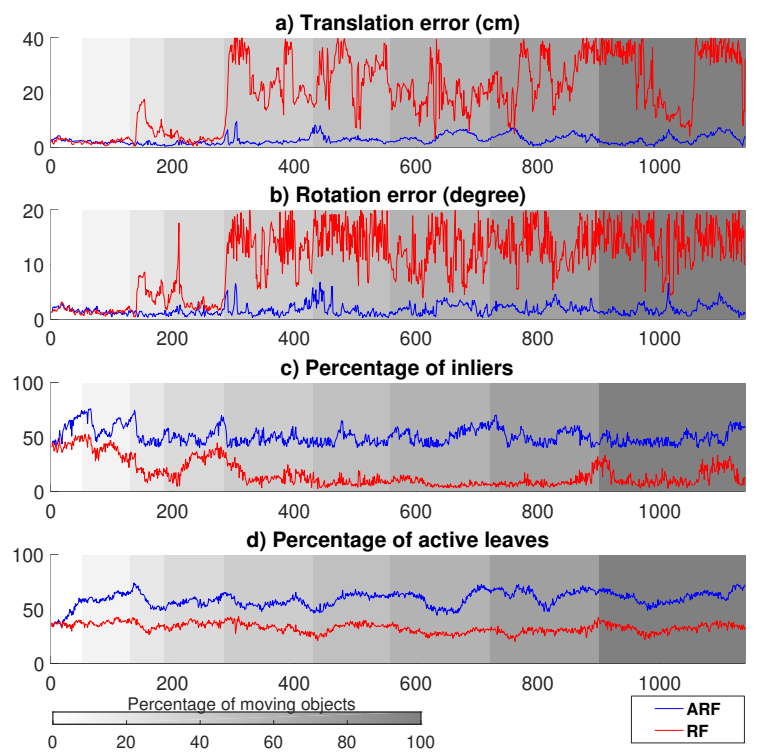

Figure 4: Detail results of our DynaLoc based the ARF (blue) and RF (red) on DynaScene-03/Seq-02. a), b) translation error in centimeter and rotation error in degree. c) the percentage of number of inliers at each frame. d) the percentage of active leaves compares to the number of leaves used at each frame for predictions. The background color present the percentage of objects in the scene that have moved since the beginning.

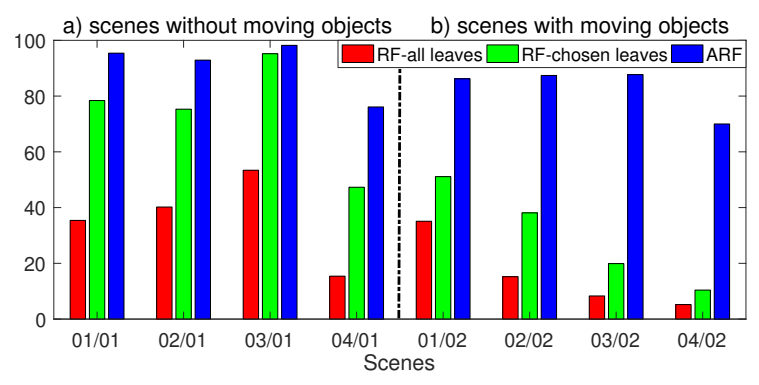

Figure 5: Comparison our DynaLoc based on the ARF (blue) to RF approaches using all leaves (red) and chosen leaves (green) with $T_{\text {var }}=0.1$ on DynaScenes dataset by measuring the percentage of test images where the pose error is below $5 \mathrm{~cm}$ and $5^{\circ}$.

again thanks to the passive leaves update step. That is why the inliers percentage remains sufficiently high, as shown in Fig. 4-c). Furthermore, although the ARF update is proceeded based on estimated results, the error accumulation is very small. The rotation and translation errors before and after movements are approximately equal as shown in Fig. 4-a,b). Therefore, our DynaLoc can handle a whole scene with gradual changes.

In Fig. 5-b), we compare the results of our DynaLoc with the RF approaches (with or without chosen leaves) on dynamic scenes. We report that the ac-

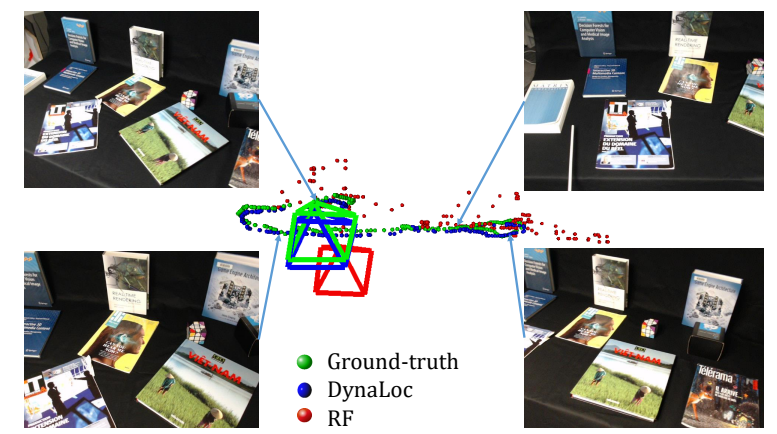

Figure 6: Comparison results between DynaLoc and RF on a dynamic sequence.

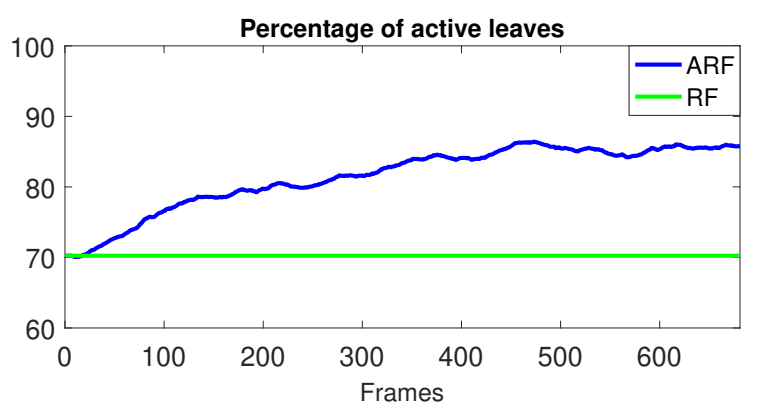

Figure 7: The percentage of active leaves in the whole regression forest at each frame for the ARF strategy (blue) and a regression forest strategy (green) on the static sequence $01 / 01$.

curacy of RF approaches drastically drops when the scene changes, whereas our DynaLoc remains stable. Fig. 6 also shows results on a part of the sequence Dyna-03/Seq-02. For this example, when some objects move gradually, the result of our DynaLoc is still accurate. Conversely, the RF approach fails completely.

Fine-tune predictive model on static scenes. Our ARF also improves the predictive model on static scenes. Indeed, Fig. 5-a) shows that the removal of uncertain leaves significantly improves accuracy (RF chosen leaves gives better results than RF all leaves). And the ARF update increases accuracy further than the static strategy (RF). This is due to our ARF finetunes the predictive model from online data. Firstly, we discard the uncertain predictive models given by passive leaves. Then, these predictive models are recalculated based on active leaves update. Fig. 7 shows the percentage of active leaves in the forest on the static sequence DynaScene-01/Seq-01 with a variance threshold $T_{\text {var }}=0.1$. Our ARF update increases the number of active leaves. 


\subsection{Comparison to state-of-the-art methods}

Baselines. We compare our method to three different RGB camera relocalization approaches: sparse feature based (Sattler et al., 2017), machine learning based (Kendall and Cipolla, 2017) and hybrid based (Brachmann et al., 2016; Brachmann et al., 2017; Brachmann and Rother, 2018; Duong et al., 2018) methods. All baselines use RGB-D images for the training phase and a single RGB image for the testing phase. We report our results on both static scenes and dynamic scenes.

\begin{tabular}{|c|c|c|}
\hline Sequence & (Duong et al., 2018) & DynaLoc \\
\hline Dyna-01/Seq-01 & $2.9 \mathrm{~cm}, 1.9^{\circ}$ & $2.6 \mathrm{~cm}, 1.7^{\circ}$ \\
\hline Dyna-02/Seq-01 & $3.2 \mathrm{~cm}, 2.6^{\circ}$ & $3.0 \mathrm{~cm}, 2.5^{\circ}$ \\
\hline Dyna-03/Seq-01 & $1.9 \mathrm{~cm}, 1.4^{\circ}$ & $1.4 \mathrm{~cm}, 1.2^{\circ}$ \\
\hline Dyna-04/Seq-01 & $4.3 \mathrm{~cm}, 3.8^{\circ}$ & $3.3 \mathrm{~cm}, 1.9^{\circ}$ \\
\hline Dyna-01/Seq-02 & $4.7 \mathrm{~cm}, 2.9^{\circ}$ & $2.2 \mathrm{~cm}, 1.6^{\circ}$ \\
\hline Dyna-02/Seq-02 & $7.2 \mathrm{~cm}, 5.1^{\circ}$ & $3.5 \mathrm{~cm}, 2.7^{\circ}$ \\
\hline Dyna-03/Seq-02 & $19.8 \mathrm{~cm}, 14.3^{\circ}$ & $2.2 \mathrm{~cm}, 1.5^{\circ}$ \\
\hline Dyna-04/Seq-02 & $25.6 \mathrm{~cm}, 20.6^{\circ}$ & $3.2 \mathrm{~cm}, 1.7^{\circ}$ \\
\hline Average & $8.7 \mathrm{~cm}, 6.6^{\circ}$ & $2.7 \mathrm{~cm}, 1.9^{\circ}$ \\
\hline
\end{tabular}

Table 2: Comparison of our method with a hybrid method based on a regression forest (Duong et al., 2018). The accuracy is evaluated by median pose errors on DynaScenes dataset.

\begin{tabular}{c|ccc|c}
\hline Scene & Active Search & PoseNet2 & DSAC++ & DynaLoc \\
\hline Chess & $0.04 \mathrm{~m}, 2.0^{\circ}$ & $0.13 \mathrm{~m}, 4.5^{\circ}$ & $\mathbf{0 . 0 2 m}, \mathbf{0 . 5}$ & $0.03 \mathrm{~m}, 1.3^{\circ}$ \\
Fire & $0.03 \mathrm{~m}, 1.5^{\circ}$ & $0.27 \mathrm{~m}, 11.3^{\circ}$ & $\mathbf{0 . 0 2 m}, \mathbf{0 . 9}^{\circ}$ & $\mathbf{0 . 0 2 m}, 1.2^{\circ}$ \\
Heads & $0.02 \mathrm{~m}, 1.5^{\circ}$ & $0.17 \mathrm{~m}, 13.0^{\circ}$ & $\mathbf{0 . 0 1 m}, \mathbf{0 . 8}^{\circ}$ & $0.02 \mathrm{~m}, 1.3^{\circ}$ \\
Office & $0.09 \mathrm{~m}, 3.6^{\circ}$ & $0.19 \mathrm{~m}, 5.6^{\circ}$ & ${\mathbf{0 . 0 3 m}, \mathbf{0 . 7}^{\circ}}^{\circ}$ & $0.04 \mathrm{~m}, 1.5^{\circ}$ \\
Pumpkin & $0.08 \mathrm{~m}, 3.1^{\circ}$ & $0.26 \mathrm{~m}, 4.8^{\circ}$ & ${\mathbf{0 . 0 4 m}, \mathbf{1 . 1}^{\circ}}^{\circ}$ & $0.05 \mathrm{~m}, 1.6^{\circ}$ \\
Kitchen & $0.07 \mathrm{~m}, 3.4^{\circ}$ & $0.23 \mathrm{~m}, 5.4^{\circ}$ & ${\mathbf{0 . 0 4 m}, \mathbf{1 . 1}^{\circ}}^{\circ}$ & $\mathbf{0 . 0 4 m}_{1} 1.7^{\circ}$ \\
Stairs & ${\mathbf{0 . 0 3 m}, 2.2^{\circ}}^{\circ}$ & $0.35 \mathrm{~m}, 12.4^{\circ}$ & $0.09 \mathrm{~m}, 2.6^{\circ}$ & $0.07 \mathrm{~m}, 1.9^{\circ}$ \\
\hline Average & $0.05 \mathrm{~m}, 2.5^{\circ}$ & $0.23 \mathrm{~m}, 8.1^{\circ}$ & ${\mathbf{0 . 0 4 m}, \mathbf{1 . 1}^{\circ}}^{\circ}$ & $\mathbf{0 . 0 4 m}, 1.5^{\circ}$
\end{tabular}

Table 3: Comparison of our method with state-of-the-art methods. The accuracy is evaluated by median pose errors on 7 scenes dataset.

\begin{tabular}{c|ccc|c}
\hline Scene & (Brachmann et al., 2016) & DSAC & (Duong et al., 2018) & DynaLoc \\
\hline Chess & $94.9 \%$ & $\mathbf{9 7 . 4 \%}$ & $73.1 \%$ & $75.2 \%$ \\
Fire & $73.5 \%$ & $71.6 \%$ & $79.5 \%$ & $\mathbf{8 2 . 3 \%}$ \\
Heads & $49.1 \%$ & $67.0 \%$ & $80.1 \%$ & $\mathbf{8 1 . 8 \%}$ \\
Office & $53.2 \%$ & $\mathbf{5 9 . 4 \%}$ & $54.5 \%$ & $58.2 \%$ \\
Pumpkin & $54.5 \%$ & $\mathbf{5 8 . 3 \%}$ & $55.1 \%$ & $56.7 \%$ \\
Kitchen & $42.2 \%$ & $42.7 \%$ & $52.2 \%$ & $\mathbf{5 4 . 5 \%}$ \\
Stairs & $20.1 \%$ & $13.4 \%$ & $41.0 \%$ & $\mathbf{4 1 . 6 \%}$ \\
\hline Average & $55.2 \%$ & $58.5 \%$ & $62.2 \%$ & $\mathbf{6 4 . 3 \%}$
\end{tabular}

Table 4: Comparison of our method with state-of-the-art methods. The accuracy is measured by the percentage of test images where the pose error is below $5 \mathrm{~cm}$ and $5^{\circ}$ on 7 scenes dataset.

DynaScenes dataset. We first compare our method to the hybrid method (Duong et al., 2018) on DynaScenes dataset. Tab. 2 shows median camera pose error for each sequence. Our results are moderately better than (Duong et al., 2018) on the sequences $01,02,03,04 / 01$ where there are challenging partial

\begin{tabular}{c|c|c}
\hline & DSAC++ & DynaLoc \\
\hline Configuration & $\begin{array}{c}\text { GPU Tesla K80 } \\
\text { Intel Xeon E5-2680 }\end{array}$ & Intel Core i7-7820HK \\
\hline Training time & $1-2$ days & $\mathbf{5 - 1 0}$ minutes \\
Testing time & $220 \mathrm{~ms}$ & $\mathbf{5 5 m s}$ (5ms for ARF update)
\end{tabular}

Table 5: Comparison of our method with DSAC++ in term of runtime. Training time per scene and testing time per image.

occlusion, illumination changes without moving objects. Both methods achieve high accuracy thanks to the use of SURF features and RANSAC algorithm. However, on the remaining sequences, (Duong et al., 2018) only obtains moderate accuracy for two scenes Dyna- $\{01,02\} /$ Seq-02 that contain respectively $30 \%$ and $60 \%$ moving objects. For the scenes Dyna$\{03,04\} /$ Seq-02 where all the objects move gradually, the accuracy of (Duong et al., 2018) drops significantly because RANSAC cannot eliminate a lot of outliers on moving objects. Inversely, our method still estimates precisely thanks to passive leaves detection and update.

7 scenes dataset. Our method is also as accurate as the state-of-the-art methods on static scenes. This is demonstrated by results on 7 scenes dataset shown in Tab. 3 and Tab. 4. Tab. 3 reports that our results clearly outperform PoseNet2 (Kendall and Cipolla, 2017) on all scenes. And they are slightly better than Active Search (Sattler et al., 2017) on this dataset except for the translation error on the stairs scene. The results also show that the accuracy of our method is approximately equal to DSAC++ (Brachmann and Rother, 2018). Tab. 4 shows the results of our method, of the hybrid methods (Brachmann et al., 2016; Brachmann et al., 2017; Duong et al., 2018) using another metric: the percentage of test images where the pose error is below $5 \mathrm{~cm}$ and $5^{\circ}$. This metric gives a better evaluation of the stably of a method on this dataset. Regarding result of each scene, our method achieves the best results on four scenes: Fire, Heads, Kitchen and Stairs. Our method achieves better accuracy than (Duong et al., 2018) on all scenes. This indicates that the update of ARF from current data improves significantly the accuracy of the initially learned model. In term of the runtime, our method is much faster than DSAC++ for both training and testing, as shown in Tab. 5.

\section{Conclusion}

In this paper, we proposed an adaptive regression forest that can update itself during the test phase with 
current observations to tackle the challenge of dynamic data. This is performed by detecting and updating passive leaves of a regression forest. We apply our adaptive regression forest to our DynaLoc, a realtime and accurate camera relocalization from a singe RGB image in dynamic scenes with moving objects. Our DynaLoc achieves high accuracy on our dynamic scenes dataset. Moreover, our method is as accurate as the state-of-the-art methods on static scenes dataset but performs more quickly both training and testing time.

\section{REFERENCES}

Alcantarilla, P. F., Yebes, J. J., Almazán, J., and Bergasa, L. M. (2012). On combining visual slam and dense scene flow to increase the robustness of localization and mapping in dynamic environments. In Robotics and Automation (ICRA), 2012 IEEE International Conference on, pages 1290-1297. IEEE.

Baatz, G., Köser, K., Chen, D., Grzeszczuk, R., and Pollefeys, M. (2012). Leveraging 3d city models for rotation invariant place-of-interest recognition. International Journal of Computer Vision, 96(3):315-334.

Bescos, B., Facil, J. M., Civera, J., and Neira, J. (2018). Dynaslam: Tracking, mapping, and inpainting in dynamic scenes. IEEE Robotics and Automation Letters, 3(4):4076-4083.

Brachmann, E., Krull, A., Nowozin, S., Shotton, J., Michel, F., Gumhold, S., and Rother, C. (2017). Dsac - differentiable ransac for camera localization. In The IEEE Conference on Computer Vision and Pattern Recognition $(C V P R)$

Brachmann, E., Michel, F., Krull, A., Ying Yang, M., Gumhold, S., et al. (2016). Uncertainty-driven 6d pose estimation of objects and scenes from a single rgb image. In Proceedings of the IEEE Conference on Computer Vision and Pattern Recognition, pages 3364-3372.

Brachmann, E. and Rother, C. (2018). Learning less is more-6d camera localization via $3 \mathrm{~d}$ surface regression. In Proc. CVPR, volume 8.

Cavallari, T., Golodetz, S., Lord, N. A., Valentin, J., Di Stefano, L., and Torr, P. H. S. (2017). On-the-fly adaptation of regression forests for online camera relocalisation. In The IEEE Conference on Computer Vision and Pattern Recognition (CVPR).

Clark, R., Wang, S., Markham, A., Trigoni, N., and Wen, H. (2017). Vidloc: A deep spatio-temporal model for 6dof video-clip relocalization. In The IEEE Conference on Computer Vision and Pattern Recognition (CVPR).

Davison, A. J., Reid, I. D., Molton, N. D., and Stasse, O. (2007). Monoslam: Real-time single camera slam. IEEE transactions on pattern analysis and machine intelligence, 29(6):1052-1067.

Duong, N.-D., Kacete, A., Soladie, C., Richard, P.-Y., and Royan, J. (2018). Accurate sparse feature regression forest learning for real-time camera relocalization. In 2018 International Conference on 3D Vision (3DV), pages 643-652. IEEE.

Engel, J., Schöps, T., and Cremers, D. (2014). Lsd-slam: Large-scale direct monocular slam. In European Conference on Computer Vision, pages 834-849. Springer.

Glocker, B., Shotton, J., Criminisi, A., and Izadi, S. (2015). Real-time rgb-d camera relocalization via randomized ferns for keyframe encoding. IEEE transactions on visualization and computer graphics, 21(5):571-583.

Guzman-Rivera, A., Kohli, P., Glocker, B., Shotton, J., Sharp, T., Fitzgibbon, A., and Izadi, S. (2014). Multioutput learning for camera relocalization. In Proceedings of the IEEE Conference on Computer Vision and Pattern Recognition, pages 1114-1121.

Hartley, R. and Zisserman, A. (2005). Multiple view geometry in computer vision. Robotica, 23(2):271-271.

Kendall, A. and Cipolla, R. (2016). Modelling uncertainty in deep learning for camera relocalization. Proceedings of the International Conference on Robotics and Automation (ICRA).

Kendall, A. and Cipolla, R. (2017). Geometric loss functions for camera pose regression with deep learning. Proceedings of the IEEE Conference on Computer Vision and Pattern Recognition.

Kendall, A., Grimes, M., and Cipolla, R. (2015). Posenet: A convolutional network for real-time 6-dof camera relocalization. In Proceedings of the IEEE International Conference on Computer Vision, pages 2938-2946.

Klein, G. and Murray, D. (2007). Parallel tracking and mapping for small ar workspaces. In Mixed and Augmented Reality, 2007. ISMAR 2007. 6th IEEE and ACM International Symposium on, pages 225-234. IEEE.

Meng, L., Chen, J., Tung, F., Little J., J., Valentin, J., and Silva, C. (2017). Backtracking regression forests for accurate camera relocalization. In IEEE/RSJ International Conference on Intelligent Robots and Systems (IROS 2017).

Mur-Artal, R., Montiel, J. M. M., and Tardos, J. D. (2015). Orb-slam: a versatile and accurate monocular slam system. IEEE Transactions on Robotics, 31(5):11471163.

Newcombe, R. A., Fox, D., and Seitz, S. M. (2015). Dynamicfusion: Reconstruction and tracking of nonrigid scenes in real-time. In Proceedings of the IEEE conference on computer vision and pattern recognition, pages 343-352.

Newcombe, R. A., Izadi, S., Hilliges, O., Molyneaux, D., Kim, D., Davison, A. J., Kohi, P., Shotton, J., Hodges, S., and Fitzgibbon, A. (2011). Kinectfusion: Realtime dense surface mapping and tracking. In Mixed and augmented reality (ISMAR), 2011 10th IEEE international symposium on, pages 127-136. IEEE.

Riazuelo, L., Montano, L., and Montiel, J. (2017). Semantic visual slam in populated environments. In $2017 \mathrm{Eu}$ ropean Conference on Mobile Robots (ECMR), pages 1-7. IEEE.

Sattler, T., Leibe, B., and Kobbelt, L. (2011). Fast imagebased localization using direct $2 \mathrm{~d}$-to-3d matching. In 
2011 International Conference on Computer Vision, pages 667-674. IEEE.

Sattler, T., Leibe, B., and Kobbelt, L. (2017). Efficient \& effective prioritized matching for large-scale imagebased localization. IEEE transactions on pattern analysis and machine intelligence, 39(9):1744-1756.

Shotton, J., Glocker, B., Zach, C., Izadi, S., Criminisi, A., and Fitzgibbon, A. (2013). Scene coordinate regression forests for camera relocalization in rgb-d images. In Proceedings of the IEEE Conference on Computer Vision and Pattern Recognition, pages 2930-2937.

Sun, Y., Liu, M., and Meng, M. Q.-H. (2017). Improving rgb-d slam in dynamic environments: A motion removal approach. Robotics and Autonomous Systems, 89:110-122.

Tan, W., Liu, H., Dong, Z., Zhang, G., and Bao, H. (2013). Robust monocular slam in dynamic environments. In Mixed and Augmented Reality (ISMAR), 2013 IEEE International Symposium on, pages 209-218. IEEE.

Tateno, K., Tombari, F., Laina, I., and Navab, N. (2017). Cnn-slam: Real-time dense monocular slam with learned depth prediction. In The IEEE Conference on Computer Vision and Pattern Recognition (CVPR).

Tsai, R. Y. and Lenz, R. K. (1988). Real time versatile robotics hand/eye calibration using $3 \mathrm{~d}$ machine vision. In Robotics and Automation, 1988. Proceedings., 1988 IEEE International Conference on, pages 554-561. IEEE.

Valentin, J., Nießner, M., Shotton, J., Fitzgibbon, A., Izadi, S., and Torr, P. H. (2015). Exploiting uncertainty in regression forests for accurate camera relocalization. In Proceedings of the IEEE Conference on Computer Vision and Pattern Recognition, pages 4400-4408.

Walch, F., Hazirbas, C., Leal-Taixe, L., Sattler, T., Hilsenbeck, S., and Cremers, D. (2017). Image-based localization using lstms for structured feature correlation. In The IEEE International Conference on Computer Vision (ICCV).

Wangsiripitak, S. and Murray, D. W. (2009). Avoiding moving outliers in visual slam by tracking moving objects. In ICRA, volume 2 , page 7 . 